\title{
An Elastic Optical Network-based Architecture for the 5G Fronthaul
}

\author{
Mateus Riva $^{1}$, Henrique Donâncio ${ }^{1}$, Felipe R. Almeida ${ }^{2}$, Gustavo B. Figueiredo ${ }^{2}$, \\ Rodrigo I. Tinini ${ }^{1}$, Roberto M. Cesar Jr. ${ }^{1}$, Daniel M. Batista ${ }^{1}$ \\ ${ }^{1}$ Departamento de Ciência da Computação \\ Instituto de Matemática e Estatística - University of São Paulo (IME-USP) \\ São Paulo - SP - Brazil \\ ${ }^{2}$ Departamento de Ciência da Computação \\ Federal University of Bahia \\ Salvador - BA - Brazil \\ \{mriva, donancio, rtinini, cesar, batista\}@ime.usp.br \\ felipera@openmailbox.org, gustavoddcc.ufba.br
}

\begin{abstract}
In $5 G$ networks, the traffic demands are expected to increase significantly. To deal with this problem, many research efforts focus on TWDMPON for the fronthaul. However, TWDM-PON suffers from the issue of wasted bandwidth when a demand is smaller than the channel. In order to avoid this inefficiency, we propose an OFDM-PON supported architecture for the Cloud Radio Access Network (C-RAN) topology to implement the technology of elastic optical networks into C-RAN. Experiments show that OFDM-PON allows for improved usage of the bandwidth (between $145 \%$ and $218 \%$ improvement), similar average wait time for requests (average difference below 7\%), and similar request loss (average difference below 2\%) in comparison to TWDM.
\end{abstract}

\section{Introduction}

With the approaching advent of $5 \mathrm{G}$ networking, mobile traffic demands are expected to rise by an astounding degree [Cisco 2016]. The current 4G Distributed Radio Access Network (DRAN) infrastructure, schematized in Figure 1 (a), cannot be expected to cope with this increase in a power- and time-efficient way, due to increasing interference and traffic demands. Therefore, new technologies are necessary in order to enable 5G networking. One of the technologies proposed is the Cloud Radio Access Network (C-RAN), which separates the antennas from the baseband processing hardware (BaseBand Units, or BBUs), grouping and centralizing BBUs into a cloud in order to reduce energy consumption, provide sharing of computing resources and improve coordinated multipoint techniques [Sawahashi et al. 2010]. This is necessary for energy-efficiently mitigating the expected increase in interference from an increasing number of devices in the same local region. In such an architecture, antenna towers are equipped with only a simple radio signal processing unit, called the Remote Radio Head (RRH). The communication between the RRHs and the BBU cloud is enabled by an interconnection network (usually optical) called fronthaul [Tanaka and Agata 2015]. This is schematized in Figure 1 (b).

Currently, several research efforts focus on Time-Wavelength Division Multiplexing in Passive Optical Networks (TWDM-PON) as the solution for the extreme traffic demands placed upon the fronthaul [Iida et al. 2013, Figueiredo et al. 2016, 

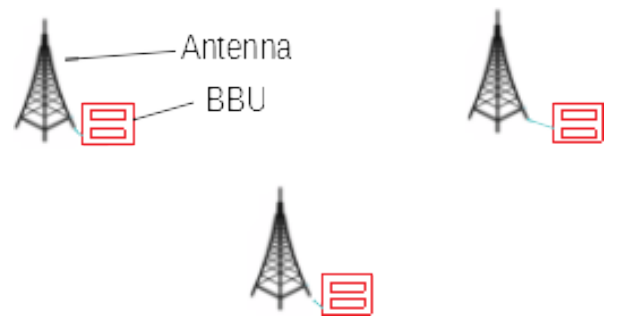

(a)

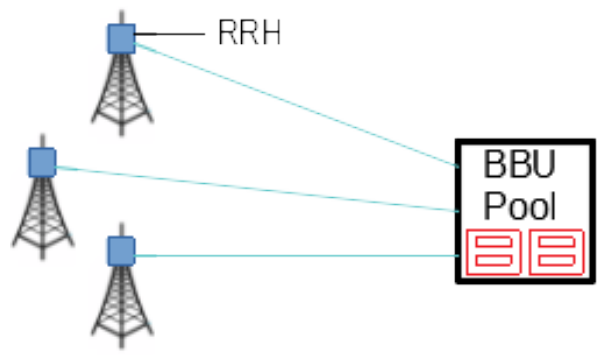

(b)

Figure 1. Schematics of the (a) DRAN topology; (b) C-RAN topology.

Wang et al. 2016], in order to improve on current techniques such as Wavelength Division Multiplexing (WDM). However, TWDM-PON suffers from the issue of wasted bandwidth when a demand is smaller than the channel.

One relatively recent development for optical bandwidth division is the Elastic Optical Network (EON) [Gerstel et al. 2012]. In standard Wavelength Division Multiplexing (WDM), the bandwidth is divided in relatively large channels and demands are allocated to channels (which should be capable of fitting the entire demand). However, if a $5 \mathrm{Gbps}$ demand was allocated into a $25 \mathrm{Gbps}$ channel, there would be a waste of $20 \mathrm{Gbps}$ of bandwidth which could be allocated to other demands. Instead of rigidly dividing the available bandwidth in "large" channels, the EON divides the bandwidth into many highgranularity slots, which are then contiguously allocated to form a channel to specifically meet a demand, with reduced waste of bandwidth. Using the technology of Orthogonal Frequency Division Multiplexing Passive Optical Networks (OFDM-PON), the concept of EON can be implemented on the C-RAN fronthaul to provide both elastic bandwidth allocation while maintaing a passive optical network infrastructure.

The use of the fronthaul bandwidth may yet be improved by the use of OFDMPON. To the best of our knowledge, no published work has yet performed an analysis of an OFDM-PON simulation in a C-RAN fronthaul. Considering the relative novelty and growing importance of the $5 \mathrm{G}$ field, as well as the established necessity of fully utilizing the fronthaul's bandwidth, we believe there is strong motivation to explore this alternative.

The objective of this paper is to investigate the possibility of improvement on the usage of the fronthaul bandwidth through the implementation of Elastic Optical Networks to implement the C-RAN transport network using the OFDM-PON technology. The contribution of this paper is the proposal of the OFDM-PON-supported CRAN fronthaul. The paper also includes an analysis of its performance when compared to the original TWDM-PON-supported proposal. Moreover, the code implemented to run the experiments represents another contribution and is publicly available at 
https://github.com/MarEe0/EON-PON-Sim under the MIT license.

This paper is organized as follows: Section 2 covers the related work available. Section 3 details the Elastic Optical Network architecture. Section 6 covers the simulation implementation and experimental setup. Section 7 displays and discusses the experimental results. Our conclusions can be found in Section 8.

\section{Related Work}

[Musumeci et al. 2016] propose the use of WDM optical transport networks as support for the fronthaul, citing the coming need for extreme bandwidth capacity. [Figueiredo et al. 2016] propose the utilization of a TWDM-PON network as support for the fronthaul. Both papers are related to the subject of the efficient usage of fronthaul bandwidth, which we tackle using Elastic Optical Networks.

A survey of spectrum management techniques for EONs was done by [Talebi et al. 2014]. Their work describes the need for Routing and Spectrum Assignment (RSA) solutions in EONs, detailing the nature of the problem. The paper also reviews many algorithms and techniques for solving the RSA problem, both online and offline. [Aibin and Walkowiak 2014] tackle the problem of dynamically routing anycast and unicast traffic in EONs, with the additional complication of considering that EONs support various modulation formats, thus adding the need to solve for "modulation" to the RSA problem. As our experiments were based on a simulation of a EON, the mechanisms proposed in these papers were studied as solutions of the RSA problem.

[Zhang et al. 2015] proposes and reviews the use of Elastic Optical Networks for connecting RRHs to the BBU cloud, demonstrating the viability of such a technology for a $5 \mathrm{G}$ network and strengthening our hypothesis of the viability of using EONs in the fronthaul of a C-RAN architecture.

[Velasco et al. 2017] propose and study the use of elastic supports for C-RAN, with particular focus on measuring gain in Operational Expenditures (OPEX) and Capital Expenditures (CAPEX). Their conclusions, obtained with an Integer Linear Programming model, point to the necessity of fine-granularity elastic networks for the C-RAN topology, and report improvements in both CAPEX and OPEX. These results show the need for further study and implementations of EONs in the C-RAN topology.

\section{Elastic Optical Networks and OFDM-PON}

Elastic Optical Networks, or alternatively Flexible Optical Networks, are a recently developed technology for bandwidth multiplexing [Gerstel et al. 2012]. It aims to improve the overall usage of the bandwidth of an optical link by diminishing the waste of bandwidth caused by the allocation of demands in large channels. This is the case in traditional fixed-grid networks such as Wavelength Division Multiplexing (WDM), or even in more recent methods such as Time-Wavelength Division Multiplexing (TWDM).

In an elastic optical network, the available bandwidth is split over several highgranularity "frequency slots", which are contiguously allocated to attend to a specific demand. For example, suppose a demand with a data rate of 6Gbps. In a traditional WDM network, with channels rated for $10 \mathrm{Gbps}$, allocating a channel for this demand would result in the waste of 4Gbps of the channel's bitrate. However, in an EON with 
slots rated for $2 \mathrm{Gbps}$, for example, only the necessary amount of contiguous slots would be allocated in order to attend to the demand, and there would be no waste of bandwidth.

In [Gerstel et al. 2012], Elastic Optical Networks are shown to always have an increase in transmission efficiency over traditional WDM networks. This increase varies from relatively insignificant, such as a $5 \%$ improvement, up to a $150 \%$ improvement. This efficiency increase depends on the nature of the network demands, the fixed WDM solution, and the elastic solution.

In OFDM technology, fine bandwidth granularity can be achieved by the transmissions and multiple modulation formats (BPSK, QPSK, and m-QAM) can be used to improve spectral efficiency. In this work, only the BPSK modulation was explored, with spectral efficiency of $1 \mathrm{~b} / \mathrm{s} / \mathrm{Hz}$, and slots of $6.25 \mathrm{GHz}$. Combining the OFDM with PON results in the OFDM-PON architecture that implements the concept of EON, but in a passive way where there is no additional processing in intermediate nodes that could incur more delay times and power consumption to the fronthaul operation.

The reason for choosing OFDM over other waveform generation techniques to implement the PON fronthaul is that a significant amount of research and technologies have been developed specifically for OFDM, which has recently been showing promise as the next high-speed solution to elastic optical networking [Talebi et al. 2014].

\section{OFDM-PON C-RAN Operation}

In our OFDM-PON C-RAN architecture, each RRH is connected to the cloud through optical fibers that are multiplexed into a optical splitter that forwards the incoming wavelengths to the cloud. The cloud implements an virtual Optical Line Terminal (OLT) that is responsible to grant transmissions to the RRHs through a Dynamic Bandwidth Allocation (DBA) algorithm. In order to provide the baseband processing to the RRHs, the OLT implements a list of virtual Line Cards (LC) that are responsible to receive the baseband signals on a specific optical frequency and forward the data to a virtual BBU (vBBU) for the baseband processing. On the transmitter side, to promote the fine granularity bandwidth allocation, the RRHs are connected to Optical Network Units (ONU) equipped with flexible transponders, that are responsible to tune the ONU transmissions to a specific frequency range to form an optical channel.

Each ONU receives the incoming traffic from the RRHs and send a transmission request to the OLT, informing its bandwidth demand. When the OLT receives the transmission request from the ONU, it will grant to the ONU a set of contiguously frequency slots that fit to the bandwidth demand of the RRHs connected to that ONU to form an optical channel and allocate to the ONU a LC that will be tuned to the same frequency of the granted frequency slots/optical channel and a vBBU. An optical channel, a LC and a vBBU can be granted to one or more ONUs, thus creating a Virtual Passive Optical Network (VPON).

In a VPON, a group of ONUs will share the same optical channel and the same vBBU to transmit and process the baseband data. With the VPON created, the OLT is responsible to schedule each transmission of the ONUs on the VPON on the time domain, providing time slots to each ONU to transmit their data through the same optical channel. If there are no frequency slots available on the fiber, the OLT can not send a grant to the ONUs and thus the transmission of one or more ONUs will be blocked. 


\section{OFDM-PON DBA algorithm}

When the OLT receives a transmission request, it executes a Dynamic Bandwidth Allocation (DBA) algorithm that is responsible to form the VPONs and provide the time slots to the transmissions. In this work we modified the interleaved polling and adaptive cycle time (IPACT) DBA algorithm to provide grants to the RRHs considering the operation of the OFDM technology. In sum, our DBA implements a procedure to find a contiguously set of frequency slots that can be allocated to fit the RRHs bandwidth demand. In other words, it implements a Spectrum Assignment (SA) algorithm. In the following, our modified IPACT DBA is explained.

When an ONU generates a request, this request is sent to a DBA assigner, located in the BBU cloud. The DBA assigner first checks if this ONU currently has a DBA assigned to it, that is, if it is already part of a VPON which is currently working on a demand. If so, then the request is discarded, and the ONU should try again later. Ordinarily, however, the ONU will not have a DBA assigned to it, and the DBA assigner will run the SA algorithm.

The SA algorithm used is a naïve sequence, allocating the first sequence of slots of the required size available. Further work could explore alternatives to this RSA solution, possibly by implementing one of the solutions available in [Talebi et al. 2014]. If slot assignment was not possible, that is, there was no contiguous sequence of free slots where the demand would fit, then the request is discarded. Otherwise, a new DBA is created and assigned to the ONU.

The recently created DBA sends a grant to the ONU. After that, it measures how long will it take for the demand to be fully attended to. After this time has passed, the DBA is destroyed and the assigned slots are freed.

This slot assignment process, along with the VPON destruction and slot release process, is schematized in Figure 2: in (a), during moment $t=1$, a new demand arrives, slots are assigned, and a VPON is created. In (b), during moment $t=2$, the previous demand is being attended to by the created VPON, and a new demand arrives, for whom slots are assigned and another VPON is created. In (c), during moment $t=3$, the first demand is finished, the created VPON is destroyed, and the slots are freed.

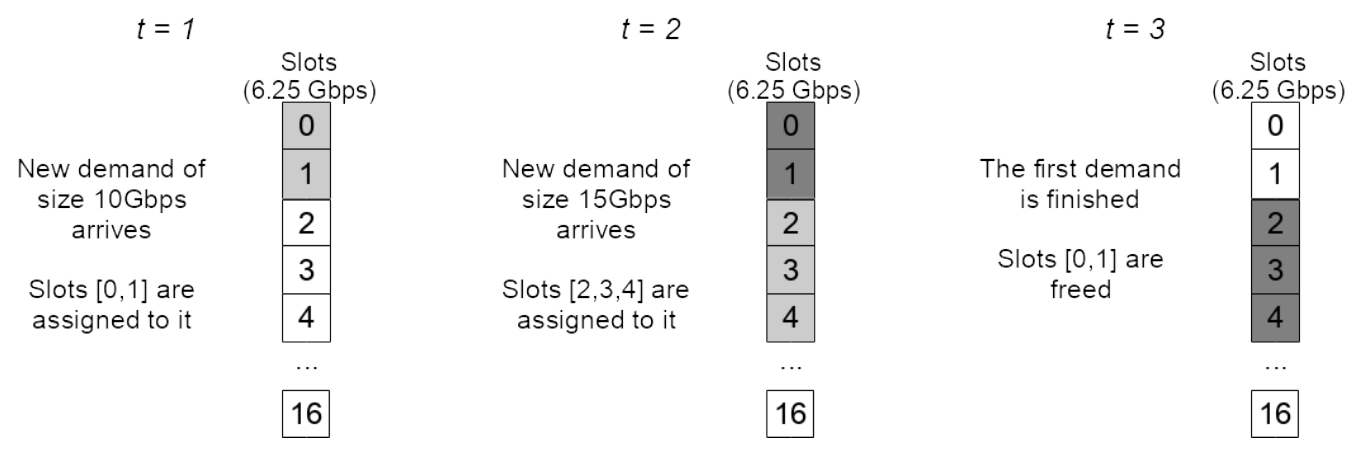

Figure 2. Schematic of the slot assignment and release process. 


\section{Experimental Setup}

\subsection{SimPy}

In order to simulate a 5G network and its demands, we used the SimPy [Sim 2016] discrete event simulator framework. SimPy was extended in order to specifically simulate a 5G network structure. This extension allows for the measurement of energy and transmission efficiency on any part of a network, including the fronthaul.

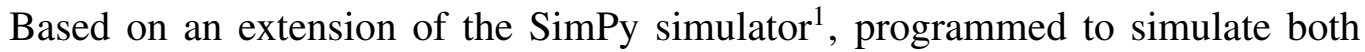
traditional C-RAN and heterogeneous C-RAN [Peng et al. 2014] topologies, we developed a simulator capable of simulating Elastic Optical Network-supported topologies ${ }^{2}$, to conduct our experiments.

\subsection{Experiments}

We aimed to simulate a C-RAN network, supported by:

- TWDM-PON;

- OFDM-based Passive EON

and compare them in terms of both energy consumption and transmission efficiency. Transmission efficiency was measured by network load over time, average packet wait time and percentage of blocked requests.

General simulation parameters are given in Table 1. Three experiments were run for both EON- and TWDM-supported simulators: each one had a different value for the total bandwidth available in the fronthaul, with total bandwidths $T=$ $\{30000,50000,100000\}$ Gbps. We will call these experiments “3”, "5" and "10", respectively; for example, the experiment "TWDM-5" refers to a simulation run with TWDM support and total bandwidth of $50000 \mathrm{Gbps}$ (which translates to five channels of size 10 Gbps).

All experiments were run for a network with $x$ RRH-ONU pairs, where $x=$ $\{4,6,8,12,16,20\}$, in order to test how the network responds to an increase in traffic generation and VPON demand. Additionally, all experiments were run 35 times with different random state seeds, and the average results obtained are displayed in Section 7.

Table 1. Experiment parameters.

Distance between nodes Simulated time Traffic Generation Size Traffic Generation Rate Channel Size
Random, uniformly distributed, total distance never exceeds $10 \mathrm{~km}$ 15 seconds

A randomly chosen CPRI option between 1 and 7A Random, uniformly distributed between $[1,2] \mathrm{s}$ $10 \mathrm{~Gb}(\mathrm{TWDM}) / 6.25 \mathrm{~Gb}$ (EON)

\footnotetext{
${ }^{1}$ Available in https://github.com/Toxad/TWDM-PON-Sim, MIT license. Last accessed on December 3, 2017.

${ }^{2}$ Available in https://github.com/MarEe0/EON-PON-Sim, MIT license. Last accessed on December 3, 2017.
} 


\section{Results and Analysis}

We report three main results from the simulation: the average wait time for a request to be fulfilled; the mean bandwidth usage during the simulation; and the percentage of lost requests (that is, the blocking probability) in the network. These results are presented in Figures 3, 4 and 5, respectively. Additionally, a summary of the results is presented in Table 2.

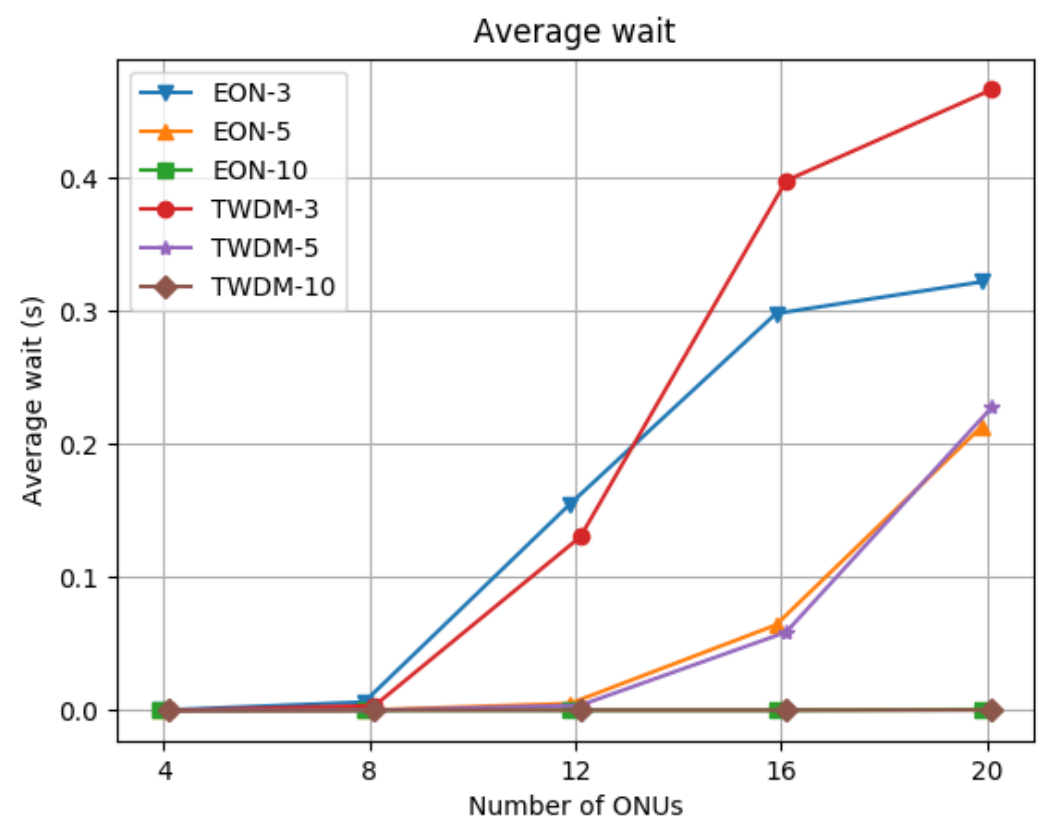

Figure 3. Average wait time for a request to be fulfilled, in seconds, in log scale. Average wait is zero when number of ONUs $=4$.

The average wait time (Figure 3) tended to be similar between TWDM- and EONsupported experiments; most discrepancies (such as the higher wait time in experiment $T=3$ for TWDM when compared to EON, when the number of ONUs is greater than 16) fall within expected simulation error.

The request blockage probability (Figure 5) was also quite similar between supports; in experiments $T=3$ and $T=5$, average differences never exceed $2 \%$. In experiment $T=10$, the EON-supported network had an average of $6 \%$ higher loss than the TWDM-supported one. Request blockage is, notably, the result with most variability between simulator executions; nevertheless, averages and standard deviations are similar between experiments for both supports.

The main difference between the EON-supported and the TWDM-supported networks was found in terms of the mean bandwidth usage (Figure 4). In terms of this metric, the EON-supported network presented gains from a $145 \%$ increase all the way up to $218 \%$, with results consistent across simulations.

These results show that, while maintaining a similar probability of request blockage and a similar average wait time for request fulfillment, the Elastic network-supported fronthaul is capable of making much more use of the available bandwidth. 


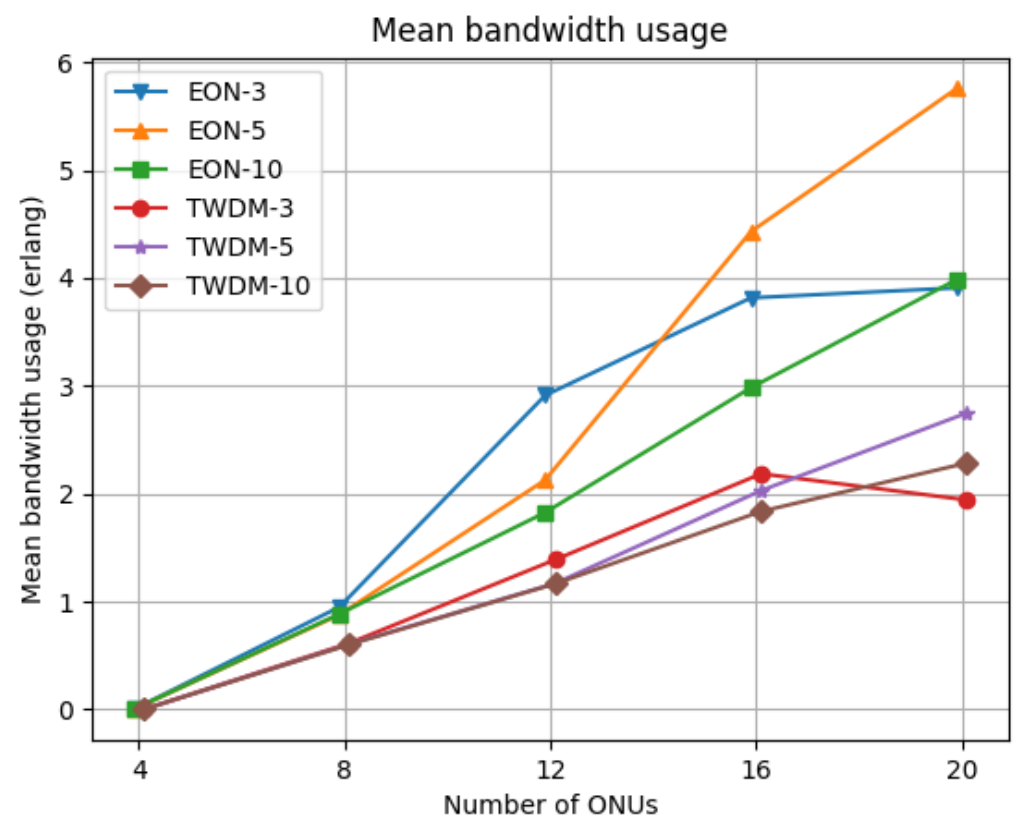

Figure 4. Mean bandwidth usage over the simulation runtime, in erlangs.

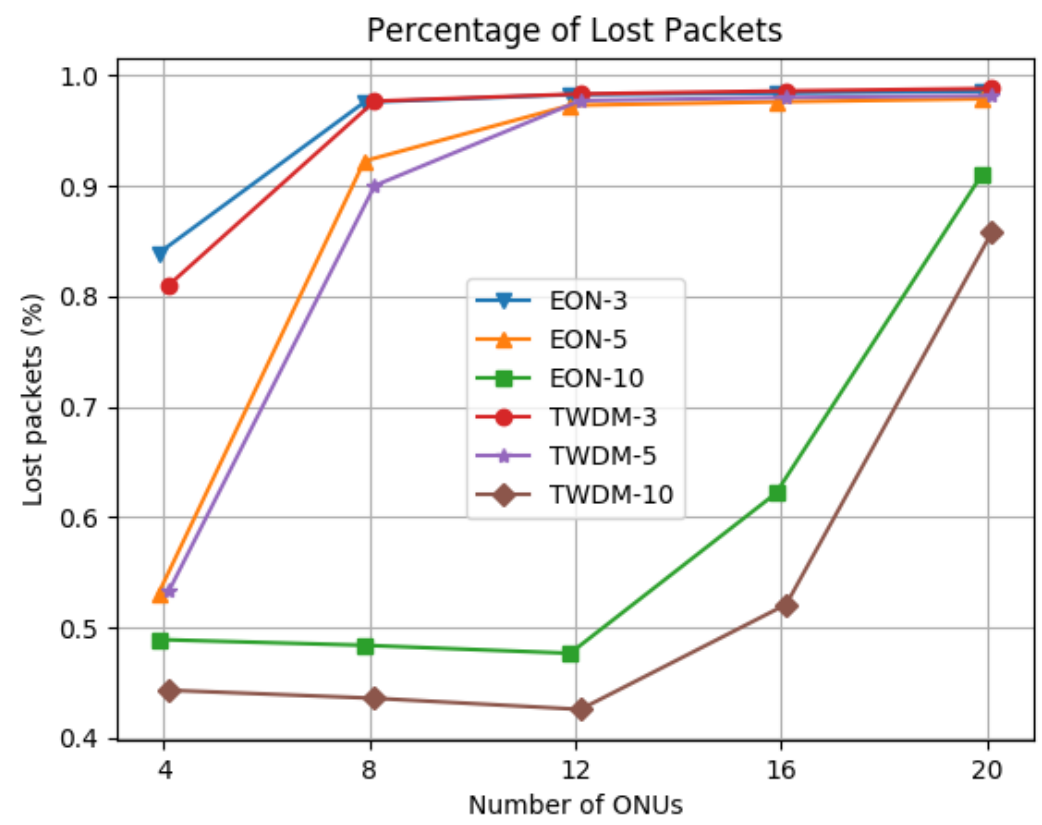

Figure 5. Percentage of lost requests.

\section{Conclusions}

We set out to propose and analyze an Elastic Optical Network-supported fronthaul for the C-RAN topology, with the expectation of improving bandwidth usage, request fulfillment speed and packet throughput over traditional fixed-grid supports. Our results show that an EON-supported fronthaul uses available bandwidth with significantly higher effi- 
Table 2. Experimental results.

\begin{tabular}{|c|c|c|c|c|c|}
\hline ONUs & $T$ & Support & Avg. wait (s) & Mean bandwidth use (e) & Ratio of lost packets \\
\hline \multirow{6}{*}{4} & \multirow{2}{*}{3} & EON & $0.0000 \pm 0.0000$ & $0.00 \pm 0.00$ & $0.839 \pm 0.343$ \\
\hline & & TWDM & $0.0000 \pm 0.0000$ & $0.00 \pm 0.00$ & $0.810 \pm 0.370$ \\
\hline & \multirow{2}{*}{5} & EON & $0.0000 \pm 0.0000$ & $0.00 \pm 0.00$ & $0.531 \pm 0.487$ \\
\hline & & TWDM & $0.0000 \pm 0.0000$ & $0.00 \pm 0.00$ & $0.533 \pm 0.489$ \\
\hline & \multirow{2}{*}{10} & EON & $0.0000 \pm 0.0000$ & $0.00 \pm 0.00$ & $0.489 \pm 0.451$ \\
\hline & & TWDM & $0.0000 \pm 0.0000$ & $0.00 \pm 0.00$ & $0.443 \pm 0.422$ \\
\hline \multirow{6}{*}{8} & \multirow{2}{*}{3} & EON & $0.0063 \pm 0.0085$ & $0.95 \pm 0.22$ & $0.976 \pm 0.019$ \\
\hline & & TWDM & $0.0036 \pm 0.0043$ & $0.62 \pm 0.08$ & $0.977 \pm 0.024$ \\
\hline & \multirow{2}{*}{5} & EON & $0.0001 \pm 0.0000$ & $0.88 \pm 0.25$ & $0.923 \pm 0.167$ \\
\hline & & TWDM & $0.0001 \pm 0.0000$ & $0.61 \pm 0.08$ & $0.900 \pm 0.229$ \\
\hline & \multirow{2}{*}{10} & EON & $0.0001 \pm 0.0000$ & $0.88 \pm 0.21$ & $0.484 \pm 0.446$ \\
\hline & & TWDM & $0.0001 \pm 0.0000$ & $0.60 \pm 0.08$ & $0.436 \pm 0.418$ \\
\hline \multirow{6}{*}{12} & \multirow{2}{*}{3} & EON & $0.1550 \pm 0.0741$ & $2.91 \pm 0.51$ & $0.983 \pm 0.008$ \\
\hline & & TWDM & $0.1303 \pm 0.0418$ & $1.39 \pm 0.20$ & $0.983 \pm 0.008$ \\
\hline & \multirow{2}{*}{5} & EON & $0.0052 \pm 0.0056$ & $2.13 \pm 0.31$ & $0.973 \pm 0.027$ \\
\hline & & TWDM & $0.0038 \pm 0.0038$ & $1.17 \pm 0.09$ & $0.977 \pm 0.021$ \\
\hline & \multirow{2}{*}{10} & EON & $0.0001 \pm 0.0000$ & $1.83 \pm 0.22$ & $0.477 \pm 0.440$ \\
\hline & & TWDM & $0.0001 \pm 0.0000$ & $1.17 \pm 0.09$ & $0.426 \pm 0.411$ \\
\hline \multirow{6}{*}{16} & \multirow{2}{*}{3} & EON & $0.2979 \pm 0.0932$ & $3.82 \pm 1.32$ & $0.984 \pm 0.006$ \\
\hline & & TWDM & $0.3977 \pm 0.0927$ & $2.19 \pm 0.41$ & $0.986 \pm 0.004$ \\
\hline & \multirow{2}{*}{5} & EON & $0.0641 \pm 0.0352$ & $4.43 \pm 0.80$ & $0.976 \pm 0.013$ \\
\hline & & TWDM & $0.0588 \pm 0.0462$ & $2.03 \pm 0.29$ & $0.980 \pm 0.010$ \\
\hline & \multirow{2}{*}{10} & EON & $0.0001 \pm 0.0000$ & $2.98 \pm 0.49$ & $0.622 \pm 0.379$ \\
\hline & & TWDM & $0.0001 \pm 0.0000$ & $1.84 \pm 0.20$ & $0.521 \pm 0.364$ \\
\hline \multirow{6}{*}{20} & \multirow{2}{*}{3} & EON & $0.3217 \pm 0.0673$ & $3.91 \pm 1.17$ & $0.986 \pm 0.005$ \\
\hline & & TWDM & $0.4661 \pm 0.0652$ & $1.94 \pm 0.39$ & $0.988 \pm 0.004$ \\
\hline & \multirow{2}{*}{5} & EON & $0.2131 \pm 0.0915$ & $5.76 \pm 0.95$ & $0.979 \pm 0.007$ \\
\hline & & TWDM & $0.2277 \pm 0.1066$ & $2.75 \pm 0.39$ & $0.982 \pm 0.005$ \\
\hline & \multirow{2}{*}{10} & EON & $0.0006 \pm 0.0009$ & $3.99 \pm 0.65$ & $0.910 \pm 0.052$ \\
\hline & & TWDM & $0.0003 \pm 0.0003$ & $2.29 \pm 0.19$ & $0.858 \pm 0.125$ \\
\hline
\end{tabular}

ciency than a TWDM-supported one, while maintaining comparable request fulfillment wait times and blocking probabilities.

Future work could investigate the advantages and potential complications of implementing EON support hardware on the fronthaul; compare the performance of the EON support against traditional fixed grid support in distinct topologies such as HCRAN [Peng et al. 2014] or Cloud-Fog RAN [Tinini et al. 2017]; and further investigate the distinct implementations of Elastic networks for the fronthaul, such as different modulations or RSA algorithms.

\section{Acknowledgements}

The authors would like to thank CAPES, the São Paulo Research Foundation (FAPESP), grants \#2017/09465-7 and \#2015/22308-2, the National Council for Scientific 
and Technological Development (CNPq), grant \#420907/2016-5 and the INCT of the Future Internet for Smart Cities funded by CNPq, proc. \#465446/2014-0, CAPES proc. \#88887.136422/2017-00, and FAPESP, proc. \#2014/50937-1.

\section{References}

(2016). SimPy: Discrete Event Simulation For Python. https://simpy . readthedocs.io/en/latest/. Accessed: 2017-09-22.

Aibin, M. and Walkowiak, K. (2014). Dynamic Routing of Anycast and Unicast Traffic in Elastic Optical Networks with Various Modulation Formats-Trade-off between Blocking Probability and Network Cost. In 2014 IEEE 15th International Conference on High Performance Switching and Routing (HPSR), pages 64-69. IEEE.

Cisco (2016). The Zettabyte Era: Trends and Analysis. https://www . cisco.com/c/en/us/solutions/collateral/service-provider/ visual-networking-index-vni/vni-hyperconnectivity-wp. html. Accessed: 2017-09-21.

Figueiredo, G. B., Wang, X., Meixner, C. C., Tornatore, M., and Mukherjee, B. (2016). Load Balancing and Latency Reduction in Multi-user CoMP over TWDM-VPONs. In 2016 IEEE International Conference on Communications (ICC), pages 1-6. IEEE.

Gerstel, O., Jinno, M., Lord, A., and Yoo, S. B. (2012). Elastic Optical Networking: A New Dawn For The Optical Layer? IEEE Communications Magazine, 50(2).

Iida, D., Kuwano, S., Kani, J.-i., and Terada, J. (2013). Dynamic TWDM-PON for Mobile Radio Access Networks. Optics Express, 21(22):26209-26218.

Musumeci, F., Bellanzon, C., Carapellese, N., Tornatore, M., Pattavina, A., and Gosselin, S. (2016). Optimal BBU Placement for 5G C-RAN Deployment Over WDM Aggregation Networks. Journal of Lightwave Technology, 34(8):1963-1970.

Peng, M., Li, Y., Jiang, J., Li, J., and Wang, C. (2014). Heterogeneous cloud Radio Access Networks: A New Perspective for Enhancing Spectral and Energy Efficiencies. IEEE Wireless Communications, 21(6):126-135.

Sawahashi, M., Kishiyama, Y., Morimoto, A., Nishikawa, D., and Tanno, M. (2010). Coordinated Multipoint Transmission/Reception Techniques for LTE-Advanced [Coordinated and Distributed MIMO]. IEEE Wireless Communications, 17(3).

Talebi, S., Alam, F., Katib, I., Khamis, M., Salama, R., and Rouskas, G. N. (2014). Spectrum Management Techniques for Elastic Optical Networks: A Survey. Optical Switching and Networking, 13:34-48.

Tanaka, K. and Agata, A. (2015). Next-generation Optical Access Networks for C-RAN. In Optical Fiber Communication Conference, pages Tu2E-1. Optical Society of America.

Tinini, R. I., Reis, L. C. M., Batista, D. M., Figueiredo, G. B., Tornatore, M., and Mukherjee, B. (2017). Optimal Placement of Virtualized BBU Processing in Hybrid CloudFog RAN over TWDM-PON. In GLOBECOM 2017 - 2017 IEEE Global Communications Conference, pages 1-6. 
Velasco, L., Castro, A., Asensio, A., Ruiz, M., Liu, G., Qin, C., Proietti, R., and Yoo, S. (2017). Meeting the Requirements to Deploy Cloud RAN Over Optical Networks. Journal of Optical Communications and Networking, 9(3):B22-B32.

Wang, X., Wang, L., Cavdar, C., Tornatore, M., Figueiredo, G. B., Chung, H. S., Lee, H. H., Park, S., and Mukherjee, B. (2016). Handover Reduction in Virtualized Cloud Radio Access Networks Using TWDM-PON Fronthaul. Journal of Optical Communications and Networking, 8(12):B124-B134.

Zhang, J., Ji, Y., Zhang, J., Gu, R., Zhao, Y., Liu, S., Xu, K., Song, M., Li, H., and Wang, X. (2015). Baseband Unit Cloud Interconnection Enabled by Flexible Grid Optical Networks with Software Defined Elasticity. 53:90-98. 